\title{
Cooperative Robot Control and Synchronization of Lagrangian Systems
}

\author{
Soon-Jo Chung and Jean-Jacques E. Slotine
}

\begin{abstract}
This article presents a simple synchronization framework that can be directly applied to cooperative control of multi-agent systems and oscillation synchronization in robotic manipulation and teleoperation. A dynamical network of multiple Lagrangian systems is constructed by adding diffusive couplings to otherwise freely moving or flying robots. The proposed decentralized tracking control law synchronizes an arbitrary number of robots into a common trajectory with global exponential convergence. The proposed strategy is much simpler than earlier work in terms of both the computational load and the required signals. Furthermore, in contrast with prior work which used simple double integrator models, the proposed method permits highly nonlinear systems and is further extended to time-delayed communications, adaptive control, partial-joint coupling, and leader-follower networks.
\end{abstract}

\section{INTRODUCTION}

Group synchronization and cooperative control are topics that are currently receiving a lot of interest in a variety of research communities. The objective of this paper is to introduce a unified synchronization framework that can be directly applied to cooperative control of multi-robot systems or vehicle formations. For example, a large swarm of robots can synchronize first to form a certain formation pattern, then track the common trajectory. In manufacturing applications, where high manipulability and maneuverability cannot be achieved by a single system [9], there has been widespread interest in cooperative manipulation schemes. A stellar formation flight interferometer is another example where precision control of relative spacecraft motions is indispensable. The proposed synchronization tracking control law can be implemented for such purposes. Other potential applications include oscillation synchronization of robotic locomotion, and tele-manipulation of robots.

The consensus problems on graph [5] and the coordination of multi-agent systems [2], [6], [7] are closely related with the synchronization problem. In particular, the use of graph theory and Laplacian produced many interesting results [2], [4], [5]. The main drawback of the aforementioned work is that they mainly deal with very simple dynamic models such as linear systems and single or double integrator models with a constant inertia matrix. Hence, most of earlier work on multi-agent coordination cannot be used for highly nonlinear systems (e.g. helicopters, spacecraft attitude dynamics, and manipulator robots). As shall be seen later, the proof of

Doctoral Student at MIT when this work was performed. Currently, assistant professor of Aerospace Engineering, Iowa State University, Ames, Iowa. s jchungealum.mit. edu.

Professor of Mechanical Engineering \& Information Sciences, Professor of Brain \& Cognitive Sciences, MIT. jjs@mit. edu . the synchronization for network systems that possess a nonlinear inertia matrix is much more involved and difficult. In addition, the mutual synchronization problem, which not only synchronizes the sub-members but also enforces them to follow a common reference trajectory, is not addressed in the consensus problems. This paper focuses on the mutual synchronization of dynamical networks consisting of highly nonlinear time-varying systems. Since the main nonlinear stability tool is contraction analysis [3], [10], we derive exact and global stability results with exponential convergence.

One recent representative work on synchronization of multi-link robots is [9]. The following difficulties can be identified. The number of variables to be estimated increases with the number of robots to be synchronized, which imposes a significant communication burden. Additionally, the feedback of estimated acceleration errors requires unnecessary information and complexity. Thus, a method to eliminate both the all-to-all coupling and the feedback of the acceleration terms is explored in this paper.

We believe our approach using contraction analysis has a clear advantage in its broad applications to a larger class of identical or nonidentical nonlinear time-varying systems even with time-delays, non-passive input-output, and complex coupling geometry including concurrent synchronization [8] and partial degrees-of-freedom coupling, while ensuring a simple decentralized coupling control law (see Fig. 1 for network structures permitted in this paper).

\section{Modeling of Multi-Robot Network}

The equations of motion for a robot with multiple joints $\left(\mathbf{q}_{i} \in \mathbb{R}^{n}\right)$ can be derived by exploiting the Euler-Lagrange equations:

$$
\mathbf{M}_{i}\left(\mathbf{q}_{i}\right) \ddot{\mathbf{q}}_{i}+\mathbf{C}_{i}\left(\mathbf{q}_{i}, \dot{\mathbf{q}}_{i}\right) \dot{\mathbf{q}}_{i}+\mathbf{g}_{i}\left(\mathbf{q}_{i}\right)=\tau_{i}
$$

where $i,(1 \leq i \leq p)$ denotes the index of robots or dynamical systems comprising a network, and $p$ is the total number of the individual elements. In addition, $\mathbf{g}_{i}\left(\mathbf{q}_{i}\right)=\frac{\partial V}{\mathbf{q}_{i}}$, and $\tau_{i}$ is a generalized force or torque acting on the $i$-th robot. Note that we define $\mathbf{C}_{i}\left(\mathbf{q}_{i}, \dot{\mathbf{q}}_{i}\right)$ such that $\left(\dot{\mathbf{M}}_{i}-2 \mathbf{C}_{i}\right)$ is skew-symmetric, and this property plays a central role in our stability analysis using contraction theory [1].

The robot system in (1) is assumed to be fully actuated. Also, the mass-inertia matrix $\mathbf{M}_{i}\left(\mathbf{q}_{i}\right)$ is uniformly positive definite.

\section{A NeW APPROACH TO SYNCHRONIZATION TRACKING CONTROL}

An exponentially stabilizing nonlinear control law which can synchronize an arbitrary number of robots to track a common reference trajectory is introduced in this section. 

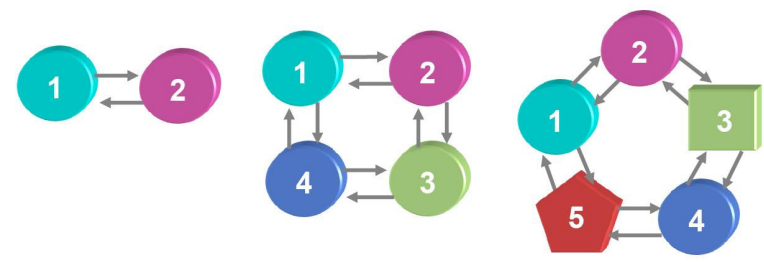

Fig. 1. Multi-agent networks of identical or nonidentical robots using local couplings. More complex geometries can also be constructed (see Sec. V-G).

\section{A. Proposed Synchronization Control Strategy}

The following tracking control law with two-way-ring symmetry is proposed for the $i$-th robot in the network consisting of $p$ identical robots (see Fig. 1):

$\tau_{i}=\mathbf{M}\left(\mathbf{q}_{i}\right) \ddot{\mathbf{q}}_{i r}+\mathbf{C}\left(\mathbf{q}_{i}, \dot{\mathbf{q}}_{i}\right) \dot{\mathbf{q}}_{i r}+\mathbf{g}\left(\mathbf{q}_{i}\right)$

$-\mathbf{K}_{1}\left(\dot{\mathbf{q}}_{i}-\dot{\mathbf{q}}_{i r}\right)+\mathbf{K}_{2}\left(\dot{\mathbf{q}}_{i-1}-\dot{\mathbf{q}}_{i-1, r}\right)+\mathbf{K}_{2}\left(\dot{\mathbf{q}}_{i+1}-\dot{\mathbf{q}}_{i+1, r}\right)$

where a positive-definite matrix $\mathbf{K}_{1} \in \mathbb{R}^{n \times n}$ is a feedback gain for the $i$-th robot, and another positive-definite matrix $\mathbf{K}_{2} \in \mathbb{R}^{n \times n}$ is a coupling gain with the adjacent members $(i-1, i+1)$. The above control law can also be applied to a network consisting of $p$ non-identical robots (Fig. 1(b)), as shall be seen in Section V-A.

The reference velocity vector, $\dot{\mathbf{q}}_{i r}$ is given by shifting the common desired velocity $\dot{\mathbf{q}}_{\mathbf{d}}$ with the position error:

$$
\dot{\mathbf{q}}_{i r}=\dot{\mathbf{q}}_{d}-\Lambda \widetilde{\mathbf{q}}_{i}=\dot{\mathbf{q}}_{d}-\Lambda\left(\mathbf{q}_{i}-\mathbf{q}_{d}\right)
$$

where $\boldsymbol{\Lambda}$ is a positive diagonal matrix.

In contrast with [9], the proposed control law requires only the coupling feedback of the most adjacent robots $(i-1$ and $i+1$ ) for exponential convergence (see Fig. 1). Note that the last ( $p$-th) robot is connected with the first robot to form a ring network as suggested in [10]. Moreover, estimates of $\ddot{\mathbf{q}}$ are no longer required.

The closed-loop dynamics using (1) and (2) become

$$
\mathbf{M}\left(\mathbf{q}_{i}\right) \dot{\mathbf{s}}_{i}+\mathbf{C}\left(\mathbf{q}_{i}, \dot{\mathbf{q}}_{i}\right) \mathbf{s}_{i}+\mathbf{K}_{1} \mathbf{s}_{i}-\mathbf{K}_{2} \mathbf{s}_{i-1}-\mathbf{K}_{2} \mathbf{s}_{i+1}=\mathbf{0}
$$

where $\mathbf{s}_{i}$ denotes the composite variable $\mathbf{s}_{i}=\dot{\mathbf{q}}_{i}-\dot{\mathbf{q}}_{i r}$.

Let us define the following $p \times p$ square matrices: $\left[\mathbf{L}_{\mathbf{A}, \mathbf{B}}^{p}\right]=$ $\left[\begin{array}{cccccc}\mathbf{A} & \mathbf{B} & \mathbf{0} & \mathbf{0} & \cdots & \mathbf{B} \\ \mathbf{B} & \mathbf{A} & \mathbf{B} & \mathbf{0} & \cdots & \mathbf{0} \\ \vdots & \ddots & \ddots & \ddots & & \vdots \\ \mathbf{0} & \cdots & \mathbf{B} & \mathbf{A} & \mathbf{B} & \mathbf{0} \\ \mathbf{B} & \cdots & \mathbf{0} & \mathbf{0} & \mathbf{B} & \mathbf{A}\end{array}\right]_{p \times p},\left[\mathbf{U}_{\mathbf{A}}^{p}\right]=\left[\begin{array}{cccc}\mathbf{A} & \mathbf{A} & \cdots & \mathbf{A} \\ \mathbf{A} & \mathbf{A} & \cdots & \mathbf{A} \\ \vdots & \vdots & \ddots & \vdots \\ \dot{\mathbf{A}} & \mathbf{A} & \cdots & \mathbf{A}\end{array}\right]_{p \times p}$

By the definition of the controller in (2), $\left[\mathbf{L}_{\mathbf{A}, \mathbf{B}}^{p}\right]$ has only three nonzero matrix elements in each row (i.e., $\mathbf{A}, \mathbf{B}, \mathbf{B})$.

Then, we can write the closed-loop dynamics in (4) in a block matrix form for $\mathbf{x}=\left(\mathbf{s}_{1}, \cdots, \mathbf{s}_{p}\right)^{T}$ :

$$
\begin{gathered}
{[\mathbf{M}] \dot{\mathbf{x}}+[\mathbf{C}] \mathbf{x}+\left(\left[\mathbf{L}_{\mathbf{K}_{1},-\mathbf{K}_{2}}^{p}\right]+\left[\mathbf{U}_{\mathbf{K}_{2}}^{p}\right]\right) \mathbf{x}=\left[\mathbf{U}_{\mathbf{K}_{2}}^{p}\right] \mathbf{x}} \\
{[\mathbf{M}]=\left[\begin{array}{ccc}
\mathbf{M}\left(\mathbf{q}_{1}\right) & \cdots & \mathbf{0} \\
\vdots & \ddots & \vdots \\
\mathbf{0} & \cdots & \mathbf{M}\left(\mathbf{q}_{p}\right)
\end{array}\right],[\mathbf{C}]=\left[\begin{array}{ccc}
\mathbf{C}\left(\mathbf{q}_{1}, \dot{\mathbf{q}}_{1}\right) & \cdots & \mathbf{0} \\
\vdots & \ddots & \vdots \\
\mathbf{0} & \cdots & \mathbf{C}\left(\mathbf{q}_{p}, \dot{\mathbf{q}}_{p}\right)
\end{array}\right] .}
\end{gathered}
$$

$\left[\mathbf{L}_{\mathbf{K}_{1},-\mathbf{K}_{2}}^{p}\right]$ can be viewed as the weighted Laplacian of the network in the context of graph theory. In other words, $\left[\mathbf{L}_{\mathbf{K}_{1},-\mathbf{K}_{2}}^{p}\right]$ indicates the connectivity with adjacent systems as well as the strength of the coupling by $\mathbf{K}_{2}$. Note that there are only three nonzero elements in each row of the matrix, which implies that there exist diffusive couplings only between adjacent members (see Fig. 1). It should be noted that the matrix $\left[\mathbf{L}_{\mathbf{K}_{1},-\mathbf{K}_{2}}^{p}\right]$ is different from a standard Laplacian matrix, which always has a zero eigenvalue; a strictly positive definite $\left[\mathbf{L}_{\mathbf{K}_{1},-\mathbf{K}_{2}}^{p}\right]$ is required for exponential convergence for the proposed control law in this paper.

We are well poised to introduce the main theorems of the present paper.

Theorem 3.1: Global Exponential Convergence to the Desired Trajectory

If $\left[\mathbf{L}_{\mathbf{K}_{1},-\mathbf{K}_{2}}^{p}\right]$ is positive definite, then every member of the network follows the desired trajectory $\mathbf{q}_{d}$ exponentially fast regardless of initial conditions.

$$
\left[\mathbf{L}_{\mathbf{K}_{1},-\mathbf{K}_{2}}^{p}\right]>0
$$

In other words, if $\mathbf{K}_{1}-2 \mathbf{K}_{2}>0$, then $\mathbf{q}_{i},(i=1,2, \cdots, p$, $p \geq 3$ ) converges to $\mathbf{q}_{d}$ exponentially fast from any initial conditions. For two-robot systems $(p=2), \mathbf{K}_{1}-\mathbf{K}_{2}>0$ needs to be true instead.

Proof: We can cancel out the $\left[\mathbf{U}_{\mathbf{K}_{2}}^{p}\right]$ matrix term in (5) to obtain

$$
[\mathbf{M}] \dot{\mathbf{x}}+[\mathbf{C}] \mathbf{x}+\left[\mathbf{L}_{\mathbf{K}_{1},-\mathbf{K}_{2}}^{p}\right] \mathbf{x}=\mathbf{0} .
$$

Consider the virtual system of $\mathbf{y}$ obtained by replacing $\mathbf{x}$ with $\mathbf{y}$ in (7).

$$
[\mathbf{M}] \dot{\mathbf{y}}+[\mathbf{C}] \mathbf{y}+\left[\mathbf{L}_{\mathbf{K}_{1},-\mathbf{K}_{2}}^{p}\right] \mathbf{y}=\mathbf{0}
$$

This virtual $\mathbf{y}$ system has two particular solutions: $\mathbf{x}=$ $\left(\mathbf{s}_{1}, \cdots, \mathbf{s}_{p}\right)^{T}$ and $\mathbf{0}$. The squared-length analysis with the positive-definite metric $[\mathbf{M}]$ yields

$$
\begin{aligned}
& \frac{d}{d t}\left(\delta \mathbf{y}^{T}[\mathbf{M}] \delta \mathbf{y}\right)=2 \delta \mathbf{y}^{T}[\mathbf{M}] \delta \dot{\mathbf{y}}+\delta \mathbf{y}^{T}[\dot{\mathbf{M}}] \delta \mathbf{y} \\
& =-2 \delta \mathbf{y}^{T}\left([\mathbf{C}] \delta \mathbf{y}+\left[\mathbf{L}_{\mathbf{K}_{1},-\mathbf{K}_{2}}^{p}\right] \delta \mathbf{y}\right)+\delta \mathbf{y}^{T}[\dot{\mathbf{M}}] \delta \mathbf{y} \\
& =-2 \delta \mathbf{y}^{T}\left[\mathbf{L}_{\mathbf{K}_{1},-\mathbf{K}_{2}}^{p}\right] \delta \mathbf{y}
\end{aligned}
$$

where we used the skew-symmetric property of $[\dot{\mathbf{M}}]-2[\mathbf{C}]$.

Accordingly, $\left[\mathbf{L}_{\mathbf{K}_{1},-\mathbf{K}_{2}}^{p}\right]>0$ will make the system contracting [3], thus all solutions of $\mathbf{y}$ converge to a single trajectory exponentially fast. This in turn indicates that the composite variable of each robot tends to zero exponentially $(\mathbf{s} \rightarrow \mathbf{0})$. By the definition of $\mathbf{s}_{i}$, the exponential convergence of $\mathbf{q}_{i}$ to the common reference trajectory $\mathbf{q}_{d}$ is proven.

The next question to be addressed is how to guarantee the synchronization of the individual dynamics.

Theorem 3.2: Synchronization of Multiple Robots Suppose the conditions in Theorem 3.1 are true, thus the individual dynamics are exponentially tracking the common desired trajectory. A swarm of $p$ robots synchronize exponentially from any initial conditions if $\exists$ diagonal matrices $\mathbf{K}_{1}>0, \mathbf{K}_{2}>0$ such that

$$
\left[\mathbf{L}_{\mathbf{K}_{1},-\mathbf{K}_{2}}^{p}\right]+\left[\mathbf{U}_{\mathbf{K}_{2}}^{p}\right]>0
$$

In addition, $\boldsymbol{\Lambda}$ is a positive diagonal matrix defining a stable composite variable $\mathbf{s}_{i}=\dot{\widetilde{\mathbf{q}}}_{i}+\Lambda \widetilde{\mathbf{q}}_{i}$. 
Proof: The proof is expanded in Section IV by separating the two different time scales of the closed-loop dynamics. As shall be seen later, multiple dynamics need not be identical to achieve stable synchronization.

It is useful to note that the above condition corresponds to $\mathbf{K}_{1}+\mathbf{K}_{2}>0$ for two-robot and three-robot networks $(p=2,3)$. A four-robot network $(p=4)$ would require $\mathbf{K}_{1}+2 \mathbf{K}_{2}>0$. We can also construct a network of multiple robots that can synchronize even with unstable tracking. In this case, the follow lemma can be used.

Lemma 3.3: Synchronization of Identical Robots with Unstable Tracking

Suppose the conditions in Theorem 3.1 are not true, thus the individual systems are exponentially unstable or indifferent. Nevertheless, a swarm of $p$ identical robots synchronize exponentially fast from any initial conditions if $\exists$ diagonal matrices $\mathbf{K}_{1}>0, \mathbf{K}_{2}$ such that

$$
\left[\mathbf{L}_{\mathbf{K}_{1},-\mathbf{K}_{2}}^{p}\right]+\left[\mathbf{U}_{\mathbf{K}_{2}}^{p}\right]>0
$$

In this case, $\boldsymbol{\Lambda}$ should be sufficiently large such that $\|\boldsymbol{\Lambda}\| \gg$ $\frac{\left\|\mathbf{K}_{1}-\mathbf{K}_{2}\right\|}{\sigma(\mathbf{M}(\mathbf{q}))}$ for $p=2$ or $\|\boldsymbol{\Lambda}\| \gg \frac{\left\|\mathbf{K}_{1}-2 \mathbf{K}_{2}\right\|}{\sigma(\mathbf{M}(\mathbf{q}))}$ for $p \geq 3$. In contrast with Theorem 3.2, the individual dynamics must be identical in the unstable tracking case.

Proof: See [1].

Note that we can render the system synchronized first, then follow the common trajectory by tuning the gains properly. For an example of a two-robot network, $\mathbf{K}_{2}>0$ ensures that the two robots synchronize faster than they follow the common desired trajectory, since $\mathbf{K}_{1}+\mathbf{K}_{2}>\mathbf{K}_{1}-\mathbf{K}_{2}$ for $\forall \mathbf{K}_{2}>0$. This indicates that there exist two different time-scales in the closed-loop systems constructed with the proposed controllers. For two-robot systems, the convergence of exponential tracking is proportional to $\mathbf{K}_{1}-\mathbf{K}_{2}$ whereas the synchronization has a convergence rate of $\mathbf{K}_{1}+\mathbf{K}_{2}$. This multi-time-scale behavior will be exploited in the subsequent sections.

\section{Proof of Exponential Synchronization}

We prove Theorem 3.2 for the exponential synchronization of multiple nonlinear dynamics in this section. Suppose that $\mathbf{M}(\mathbf{q})$ remains constant, thereby making $\mathbf{C}(\mathbf{q}, \dot{\mathbf{q}})$ zero. Then, we can easily prove $\mathbf{s}_{1}$ and $\mathbf{s}_{2}$ tend to each other from

$$
\begin{aligned}
& \mathbf{M} \dot{\mathbf{s}}_{1}+\left(\mathbf{K}_{1}+\mathbf{K}_{2}\right) \mathbf{s}_{1}=\mathbf{K}_{2}\left(\mathbf{s}_{1}+\mathbf{s}_{2}\right) \\
& \mathbf{M} \dot{\mathbf{s}}_{2}+\left(\mathbf{K}_{1}+\mathbf{K}_{2}\right) \mathbf{s}_{2}=\mathbf{K}_{2}\left(\mathbf{s}_{1}+\mathbf{s}_{2}\right)
\end{aligned}
$$

Since the virtual system

$$
\mathbf{M} \dot{\mathbf{y}}+\left(\mathbf{K}_{1}+\mathbf{K}_{2}\right) \mathbf{y}=\mathbf{K}_{2}\left(\mathbf{s}_{1}+\mathbf{s}_{2}\right)
$$

is contracting with $\mathbf{K}_{1}+\mathbf{K}_{2}>0$. Hence, its particular solutions $\mathbf{s}_{1}$ and $\mathbf{s}_{2}$ tend to each other exponentially fast according to contraction theory [3]. Without loss of generality, this result can easily be extended to arbitrarily large networks. The synchronization of a large network with a constant metric is already discussed in [10] using contraction analysis.

We now turn to a much more difficult problem focused on the synchronization of two robots with non-constant nonlinear metrics $\left(\mathbf{M}\left(\mathbf{q}_{1}\right) \neq \mathbf{M}\left(\mathbf{q}_{2}\right)\right)$.

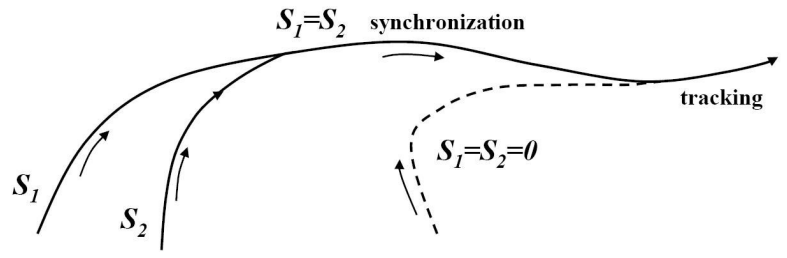

Fig. 2. Multiple timescales of synchronization (faster) and tracking (slower). The dashed line indicates the desired trajectory. Arrows indicate increasing time.

\section{A. Contraction with Two Time-Scales}

Recall the closed-loop dynamics given in (5):

$$
[\mathbf{M}] \dot{\mathbf{x}}+[\mathbf{C}] \mathbf{x}+\left[\mathbf{L}_{\mathbf{K}_{1},-\mathbf{K}_{2}}^{p}\right] \mathbf{x}=\mathbf{0}
$$

Since $\left[\mathbf{L}_{\mathbf{K}_{1},-\mathbf{K}_{2}}^{p}\right]$ is a real symmetric matrix, we can perform the spectral decomposition. This is a special case of the concurrent synchronization [8] that corresponds to convergence to a flow invariant subspace (the eigenspace).

$$
\left[\mathbf{L}_{\mathbf{K}_{1},-\mathbf{K}_{2}}^{p}\right]=\mathbf{V D V}^{T}, \quad \mathbf{V}^{T}\left[\mathbf{L}_{\mathbf{K}_{1},-\mathbf{K}_{2}}^{p}\right] \mathbf{V}=\mathbf{D}
$$

where $\mathbf{D}$ is a block diagonal matrix and $\mathbf{V}^{T} \mathbf{V}=\mathbf{V} \mathbf{V}^{T}=\mathbf{I}$. The symmetry of $\left[\mathbf{L}_{\mathbf{K}_{1},-\mathbf{K}_{2}}^{p}\right]$ gives rise to real eigenvalues and orthogonal eigenvectors.

Pre-multiplying (12) by $\mathbf{V}^{T}$ and setting $\mathbf{x}=\mathbf{V V}^{T} \mathbf{x}$ result in

$$
\begin{aligned}
\left(\mathbf{V}^{T}[\mathbf{M}] \mathbf{V}\right) \mathbf{V}^{T} \dot{\mathbf{x}} & +\left(\mathbf{V}^{T}[\mathbf{C}] \mathbf{V}\right) \mathbf{V}^{T} \mathbf{x} \\
& +\left(\mathbf{V}^{T}\left[\mathbf{L}_{\mathbf{K}_{1},-\mathbf{K}_{2}}^{p}\right] \mathbf{V}\right) \mathbf{V}^{T} \mathbf{x}=\mathbf{0}
\end{aligned}
$$

By setting $\mathbf{V}^{T} \mathbf{x}=\mathbf{z}$, (14) becomes

$$
\left(\mathbf{V}^{T}[\mathbf{M}] \mathbf{V}\right) \dot{\mathbf{z}}+\left(\mathbf{V}^{T}[\mathbf{C}] \mathbf{V}\right) \mathbf{z}+\mathbf{D z}=\mathbf{0}
$$

Then, we can develop the squared-length analysis. Notice that $\left(\mathbf{V}^{T}[\mathbf{M}] \mathbf{V}\right)$ is always symmetric positive definite since $[\mathbf{M}]$ is symmetric positive definite. For the case of a twoagent network, we can easily verify that

$$
\mathbf{V}^{T}[\mathbf{M}] \mathbf{V}=\left[\begin{array}{ll}
\frac{\mathbf{M}_{1}+\mathbf{M}_{2}}{2} & \frac{\mathbf{M}_{1}-\mathbf{M}_{2}}{2} \\
\frac{\mathbf{M}_{1}-\mathbf{M}_{2}}{2} & \frac{\mathbf{M}_{1}+\mathbf{M}_{2}}{2}
\end{array}\right], \mathbf{V}=\left[\begin{array}{cc}
\frac{1}{\sqrt{2}} \mathbf{I} & \frac{1}{\sqrt{2}} \mathbf{I} \\
\frac{1}{\sqrt{2}} \mathbf{I} & -\frac{1}{\sqrt{2}} \mathbf{I}
\end{array}\right],
$$

and $\mathbf{D}=\operatorname{diag}\left(\mathbf{K}_{1}-\mathbf{K}_{2}, \mathbf{K}_{1}+\mathbf{K}_{2}\right)$ with $\mathbf{M}_{i}=\mathbf{M}\left(\mathbf{q}_{i}\right)$.

As discussed earlier, the convergence rate of exponential tracking to the desired trajectory is proportional to $\mathbf{K}_{1}-$ $\mathbf{K}_{2}$ whereas the synchronization counterpart has the faster convergence of $\mathbf{K}_{1}+\mathbf{K}_{2}$.

$$
\mathbf{K}_{1}+\mathbf{K}_{2}>\mathbf{K}_{1}-\mathbf{K}_{2}, \forall \mathbf{K}_{2}>0
$$

This multi timescale behavior is graphically illustrated in Fig. 2. The figure ${ }^{1}$ depicts that $\mathbf{s}_{1}$ and $\mathbf{s}_{2}$ synchronize first, then they converge to the desired trajectory while staying together. This observation motivates separation of the two different time scales, namely $\mathbf{K}_{1}+\mathbf{K}_{2}$ and $\mathbf{K}_{1}-\mathbf{K}_{2}$. Intuitively, for sufficiently small $\mathbf{M}_{1}-\mathbf{M}_{2}$, the above equation

\footnotetext{
${ }^{1}$ The picture is slightly exaggerated because $\mathbf{s}_{1}$ and $\mathbf{s}_{2}$ appear overlapped when they synchronize. Strictly speaking, their difference is decreasing exponentially to zero but they will never be the same unless time tends to infinity.
} 
indicates that $\mathbf{s}_{p}$ has the convergence rate proportional to $\lambda\left(\mathbf{K}_{1}-\mathbf{K}_{2}\right)$ whereas $\mathbf{s}_{m}$ has $\lambda\left(\mathbf{K}_{1}+\mathbf{K}_{2}\right)$.

Consider the virtual system of $\mathbf{y}=\left(\mathbf{y}_{1}, \mathbf{y}_{2}\right)^{T}$ which has two particular solutions: $\left(\mathbf{y}_{1}=\mathbf{s}_{1}+\mathbf{s}_{2}, \mathbf{y}_{2}=\mathbf{s}_{1}-\mathbf{s}_{2}\right)^{T}$ and $\left(\mathbf{y}_{1}=\mathbf{0}, \mathbf{y}_{2}=\mathbf{0}\right)^{T}$

$$
\left(\mathbf{V}^{T}[\mathbf{M}] \mathbf{V}\right) \dot{\mathbf{y}}+\left(\mathbf{V}^{T}[\mathbf{C}] \mathbf{V}\right) \mathbf{y}+\mathbf{D y}=\mathbf{0}
$$

For $\mathbf{K}_{2}>0$ and $\mathbf{K}_{1}-\mathbf{K}_{2}>0$, which also lead to $\mathbf{K}_{1}+$ $\mathbf{K}_{2}>\mathbf{K}_{1}-\mathbf{K}_{2}$, we can show that the above virtual system is contracting. We take the symmetric block matrix $\mathbf{V}^{T}[\mathbf{M}] \mathbf{V}$ as our contraction metric.

Performing the squared-length analysis with respect to this metric yields

$$
\begin{aligned}
\frac{d}{d t}\left(\begin{array}{l}
\delta \mathbf{y}_{1} \\
\delta \mathbf{y}_{2}
\end{array}\right)^{T} & {\left[\begin{array}{ll}
\frac{\mathbf{M}_{1}+\mathbf{M}_{2}}{2} & \frac{\mathbf{M}_{1}-\mathbf{M}_{2}}{2} \\
\frac{\mathbf{M}_{1}-\mathbf{M}_{2}}{2} & \frac{\mathbf{M}_{1}+\mathbf{M}_{2}}{2}
\end{array}\right]\left(\begin{array}{l}
\delta \mathbf{y}_{1} \\
\delta \mathbf{y}_{2}
\end{array}\right) } \\
& =-2\left(\begin{array}{l}
\delta \mathbf{y}_{1} \\
\delta \mathbf{y}_{2}
\end{array}\right)^{T}\left[\begin{array}{cc}
\mathbf{K}_{1}-\mathbf{K}_{2} & \mathbf{0} \\
\mathbf{0} & \mathbf{K}_{1}+\mathbf{K}_{2}
\end{array}\right]\left(\begin{array}{l}
\delta \mathbf{y}_{1} \\
\delta \mathbf{y}_{2}
\end{array}\right)
\end{aligned}
$$

where we used the skew-symmetric property of $\dot{\mathbf{M}}-2 \mathbf{C}$.

We can recall the stability analysis of the trajectory tracking, depending on the sign of $\mathbf{K}_{1}-\mathbf{K}_{2}$ in Theorem 3.1. In the following, three possible cases are classified by the sign of $\mathbf{K}_{1}-\mathbf{K}_{2}$, and discussed respectively. Namely, stable tracking with $\mathbf{K}_{1}-\mathbf{K}_{2}>0$; indifferent tracking with $\mathbf{K}_{1}-\mathbf{K}_{2}=\mathbf{0}$; unstable tracking with $\mathbf{K}_{1}-\mathbf{K}_{2}<0$.

1) Case I: $\mathbf{K}_{1}-\mathbf{K}_{2}>0$ : If $\mathbf{K}_{1}+\mathbf{K}_{2}>\mathbf{K}_{1}-\mathbf{K}_{2}>0$, the rate of the virtual length in (18) is uniformly negative definite. Consequently, the combined virtual system in (17) is contracting. In other words, $\delta \mathbf{y}_{1}, \delta \mathbf{y}_{2} \rightarrow \mathbf{0}$ exponentially fast. This in turn implies all solutions of $\mathbf{y}_{1}$ and $\mathbf{y}_{2}$ tend to the single trajectory. As a result, $\mathbf{s}_{p}=\mathbf{s}_{1}+\mathbf{s}_{2}$ and $\mathbf{s}_{m}=$ $\mathbf{s}_{1}-\mathbf{s}_{2}$ tend to zero exponentially. It is straightforward to show that $\mathbf{s}_{m} \rightarrow 0$ also hierarchically makes $\mathbf{q}_{1}$ tend to $\mathbf{q}_{2}$ exponentially. From the definition of the composite variables in (4), we can find the following contracting dynamics,

$$
\left(\dot{\mathbf{q}}_{1}-\dot{\mathbf{q}}_{2}\right)+\boldsymbol{\Lambda}\left(\mathbf{q}_{1}-\mathbf{q}_{2}\right)=\mathbf{s}_{m}
$$

Note that $\dot{\mathbf{y}}+\boldsymbol{\Lambda} \mathbf{y}=0$ is contracting with $\boldsymbol{\Lambda}>0$. Consequently, $\boldsymbol{\Lambda}>0$ and $\mathbf{s}_{m} \rightarrow \mathbf{0}$ make $\mathbf{q}_{1} \rightarrow \mathbf{q}_{2}$ exponentially fast. This also implies that the diagonal terms of the metric, $\frac{\mathrm{M}\left(\mathbf{q}_{1}\right)-\mathrm{M}\left(\mathbf{q}_{2}\right)}{2}$ tend to zero exponentially, thereby eliminating the coupling of the inertia term.

$$
\left[\begin{array}{ll}
\frac{\mathbf{M}_{1}+\mathbf{M}_{2}}{2} & \frac{\mathbf{M}_{1}-\mathbf{M}_{2}}{2} \mathbf{M}_{1}-\mathbf{M}_{2} \\
\frac{\mathbf{M}_{1}+\mathbf{M}_{2}}{2}
\end{array}\right] \rightarrow\left[\begin{array}{cc}
\frac{\mathbf{M}\left(\mathbf{q}_{1}\right)+\mathbf{M}\left(\mathbf{q}_{2}\right)}{2} & \mathbf{0} \\
\mathbf{0} & \frac{\mathbf{M}\left(\mathbf{q}_{1}\right)+\mathbf{M}\left(\mathbf{q}_{2}\right)}{2}
\end{array}\right]
$$

This in turn implies that the convergence rate of tracking, $\left(\delta \mathbf{y}_{1}\right)$ is proportional to $\mathbf{K}_{1}-\mathbf{K}_{2}$ while the synchronization, $\left(\delta \mathbf{y}_{2}\right)$ occurs at a faster convergence rate, $\mathbf{K}_{1}+\mathbf{K}_{2}$. This completes the proof of Theorem 3.2.

2) Case II: $\mathbf{K}_{1}-\mathbf{K}_{2}=\mathbf{0}$ : We can also consider a case with $\mathbf{K}_{1}-\mathbf{K}_{2}=\mathbf{0}$, which fails the exponential stability condition in Theorem 3.1. The combined virtual system per se is then semi-contracting ([3]) since the squared-length analysis in (18) yields the negative semi-definite matrix:

$$
\dot{V}=\left(\begin{array}{l}
\delta \mathbf{y}_{1} \\
\delta \mathbf{y}_{2}
\end{array}\right)^{T}\left[\begin{array}{cc}
\mathbf{0} & \mathbf{0} \\
\mathbf{0} & -2\left(\mathbf{K}_{1}+\mathbf{K}_{2}\right)
\end{array}\right]\left(\begin{array}{l}
\delta \mathbf{y}_{1} \\
\delta \mathbf{y}_{2}
\end{array}\right) \leq 0
$$

While $\delta \mathbf{y}_{1}$, representing the tracking dynamics, remains in a finite ball due to $\mathbf{K}_{1}-\mathbf{K}_{2}=0, \delta \mathbf{y}_{2}$ tends to zero exponentially due to $-2\left(\mathbf{K}_{1}+\mathbf{K}_{2}\right)<0$. This result can be proven as follows. $\dot{V}$ is uniformly continuous since bounded $\delta \dot{\mathbf{y}}_{2}$ from (17) leads to bounded $\ddot{V}$ from $\ddot{V}=-4 \delta \mathbf{y}_{2}^{T}\left(\mathbf{K}_{1}+\right.$ $\left.\mathbf{K}_{2}\right) \delta \dot{\mathbf{y}}_{2}$. Due to $\dot{V} \leq 0$, the use of Barbalat's lemma verifies that $\dot{V} \rightarrow 0$ as $t \rightarrow \infty$. This implies that $\delta \mathbf{y}_{2}$ tends to zero asymptotically fast. This will eventually decouple the metric matrix with $\boldsymbol{\Lambda}>0$, as seen in (20), since $\mathbf{M}_{1}-\mathbf{M}_{2}$ tends to zero simultaneously as $\mathbf{q}_{1} \rightarrow \mathbf{q}_{2}$. As a result, when $\mathbf{M}_{1}-\mathbf{M}_{2}$ is sufficiently close to zero, the convergence of $\delta \mathbf{y}_{2} \rightarrow 0$ turns exponential.

3) Case III: $\mathbf{K}_{1}-\mathbf{K}_{2}<0$ : Consider a case when the individual tracking system is unstable with $\mathbf{K}_{1}-\mathbf{K}_{2}<0$. This case warrants further discussions. We refer the readers to [1] for details. In essence, we can show the synchronization can occur fast enough to overcome the tracking instability.

\section{B. Generalization}

We can extend the method in this section to arbitrarily large networks. For example, a network of three robots has the following $\mathbf{V}$ whose columns are orthonormal eigenvectors of $\left[\mathbf{L}_{\mathbf{K}_{1},-\mathbf{K}_{2}}^{p=3}\right]: \mathbf{V}=$ $\left[\begin{array}{ccc}-\frac{1}{\sqrt{3}} \mathbf{I} & -\frac{2}{\sqrt{6}} \mathbf{I} & \mathbf{0} \\ -\frac{1}{\sqrt{3}} \mathbf{I} & \frac{1}{\sqrt{6}} \mathbf{I} & -\frac{1}{\sqrt{2}} \mathbf{I} \\ -\frac{1}{\sqrt{3}} \mathbf{I} & \frac{1}{\sqrt{6}} \mathbf{I} & \frac{1}{\sqrt{2}} \mathbf{I}\end{array}\right]$. The diagonal matrix $\mathbf{D}$ is also computed as diag $\left(\mathbf{K}_{1}-2 \mathbf{K}_{2}, \mathbf{K}_{1}+\mathbf{K}_{2}, \mathbf{K}_{1}+\mathbf{K}_{2}\right)$. See [1] for further details.

\section{EXTENSIONS AND EXAMPLES}

Let us examine the effectiveness of the proposed control law in a variety of nonlinear dynamics networks.

\section{A. Synchronization of Non-Identical Robots}

Notice that the proposed tracking and synchronization control law in (2) can easily be applied to a network consisting of heterogenous robots in (1). For example, the $\mathbf{M}_{1}, \mathbf{M}_{2}$, $\mathbf{C}_{1}$, and $\mathbf{C}_{2}$ notations used in the previous sections can be interpreted as $\mathbf{M}_{1}=\mathbf{M}_{1}\left(\mathbf{q}_{1}\right)$ and $\mathbf{M}_{2}=\mathbf{M}_{2}\left(\mathbf{q}_{2}\right)$ with $\mathbf{M}_{1}(\cdot) \neq \mathbf{M}_{2}(\cdot)$ (the same for the $\mathbf{C}$ matrices). However, the synchronization with unstable tracking (Lemma 3.3) is no longer true for the case of non-identical robots since $\mathbf{q}_{1}=\mathbf{q}_{2}$ does not cancel $\mathbf{M}_{1}-\mathbf{M}_{2}$ in the metric matrix in (18).

\section{B. Tracking Synchronization of Four Robots}

The four identical 3-DOF robots at some arbitrary initial conditions, are driven to synchronize as well as to track the following desired trajectory (Fig. 3). The dynamics modeling of the 3-DOF robot is based upon the frictionless double inverted pendulum robot on a cart [1]. Note that this simulation fully considers the nonlinear rotational dynamics of the robots, as opposed to some earlier work on multiagent coordination [5], [6]. Fig. 4 represents a time history plot of Fig. 3. The control gains are selected such that the corresponding tracking gain $\mathbf{K}_{1}-2 \mathbf{K}_{2}=0.2$ is smaller than the synchronization gain $\mathbf{K}_{1}+2 \mathbf{K}_{2}=1.8$; the robots synchronize first, and then converge to the reference trajectory together. 


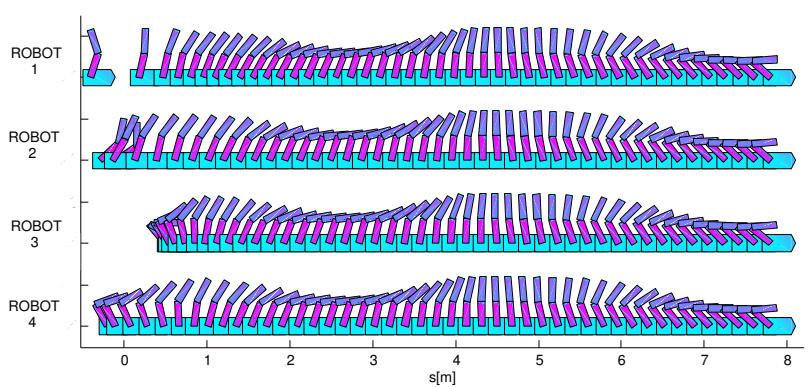

Fig. 3. Simulation results of the four-robot network
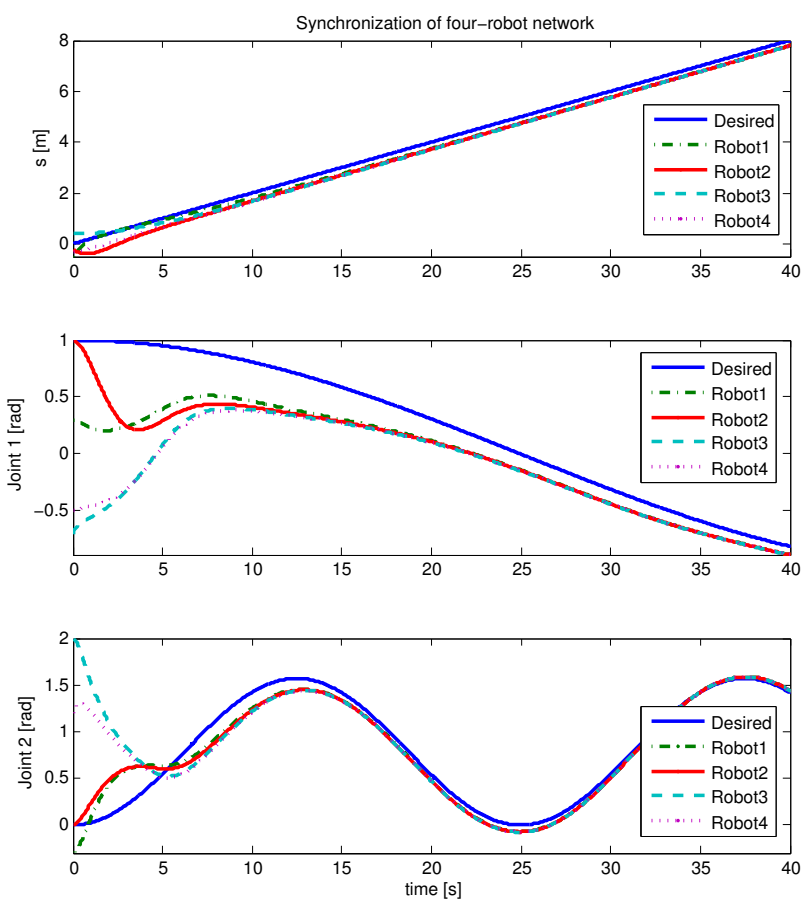

Fig. 4. Four identical robots: time history of the states

\section{Synchronization with Partial DOF Coupling}

We now consider multiple dynamics with partially coupled variables. We can assume that only the lower joint is coupled in a two-robot system having two joint variables with $\mathbf{q}=$ $\left(x_{1}, x_{2}\right)^{T}$ for $(i=1, j=2)$ or $(i=2, j=1)$ :

$$
\begin{aligned}
\tau_{i}=\mathbf{M}\left(\mathbf{q}_{i}\right) \ddot{\mathbf{q}}_{i r}+\mathbf{C}\left(\mathbf{q}_{i}, \dot{\mathbf{q}}_{i}\right) \dot{\mathbf{q}}_{i r}+\mathbf{g}\left(\mathbf{q}_{i}\right) & \\
& -\mathbf{K}_{1} \mathbf{s}_{i}+\mathbf{K}_{2}\left(\begin{array}{c}
\dot{\tilde{x}}_{1} \\
0
\end{array}\right)_{\mathbf{q}_{j}}+\mathbf{K}_{2} \boldsymbol{\Lambda}\left(\begin{array}{c}
\widetilde{x}_{1} \\
0
\end{array}\right)_{\mathbf{q}_{j}}
\end{aligned}
$$

It is straightforward to prove that Theorems 3.1 and 3.2 still hold for positive diagonal $\mathbf{K}_{1}$ and $\mathbf{K}_{2}$. This is because $\left(\mathbf{K}_{1}+\mathbf{K}_{2}\left[\begin{array}{ll}1 & 0 \\ 0 & 0\end{array}\right]\right)$ and $\left(\mathbf{K}_{1}-\mathbf{K}_{2}\left[\begin{array}{ll}1 & 0 \\ 0 & 0\end{array}\right]\right)$ are still uniformly positive definite, enabling exponential synchronization and exponential convergence to the desired trajectory, respectively. Hence, we did not break any assumptions in the proof of Theorem 3.2.

\section{Effects of Transmission Delays}

Extending [11], the proposed synchronization coupling control law in (2) is proven to synchronize multiple robots as well as to track the common trajectory, regardless of time delays in the communication. Consider two two-link manipulators transmitting their state information to each other via time-delayed transmission channels. $T_{12}$ is a positive constant denoting the time delay in the communication from the first robot to the second robot. $T_{21}$ denotes the delay from the second robot to the first robot. Similar to [11], we can modify our original Lagrangian systems consisting of two identical robots as follows

$$
\begin{aligned}
& \mathbf{M}\left(\mathbf{q}_{1}\right) \dot{\mathbf{s}}_{1}+\mathbf{C}\left(\mathbf{q}_{1}, \dot{\mathbf{q}}_{1}\right) \mathbf{s}_{1}+\mathbf{K}_{1} \mathbf{s}_{1}-\mathbf{K}_{2} \mathbf{s}_{2}\left(t-T_{21}\right)=\mathbf{0} \\
& \mathbf{M}\left(\mathbf{q}_{2}\right) \dot{\mathbf{s}}_{2}+\mathbf{C}\left(\mathbf{q}_{2}, \dot{\mathbf{q}}_{2}\right) \mathbf{s}_{2}+\mathbf{K}_{1} \mathbf{s}_{2}-\mathbf{K}_{2} \mathbf{s}_{1}\left(t-T_{12}\right)=\mathbf{0}
\end{aligned}
$$

which can be shown to be asymptotically contracting using the following differential length similar to [11]:

$$
V=\frac{1}{2} \delta \mathbf{s}_{1}^{T} \mathbf{M}\left(\mathbf{q}_{1}\right) \delta \mathbf{s}_{1}+\frac{1}{2} \delta \mathbf{s}_{2}^{T} \mathbf{M}\left(\mathbf{q}_{2}\right) \delta \mathbf{s}_{2}+\frac{1}{2} V_{1,2}
$$

where

$$
\begin{aligned}
V_{1,2}= & \int_{t-T_{12}}^{t} \delta \mathbf{v}_{12}^{T} \delta \mathbf{v}_{12} d \epsilon+\int_{t-T_{21}}^{t} \delta \mathbf{v}_{21}^{T} \delta \mathbf{v}_{21} d \epsilon \\
& -\int_{-T_{12}}^{0} \delta \mathbf{v}_{12}^{T} \delta \mathbf{v}_{12} d \epsilon-\int_{-T_{21}}^{0} \delta \mathbf{v}_{21}^{T} \delta \mathbf{v}_{21} d \epsilon
\end{aligned}
$$

In conclusion, the robot network systems, individually contracting (exponentially converging) and interacting through time-delayed diffusion-like coupling are asymptotically contracting regardless of the values of the time delays.

\section{E. Adaptive Synchronization}

Consider the following adaptive control law, which has the same local coupling structure as the proposed control law in (2):

$$
\begin{aligned}
\tau_{i} & =\mathbf{Y}_{i} \hat{\mathbf{a}}_{i}-\mathbf{K}_{1} \mathbf{s}_{i}+\mathbf{K}_{2} \mathbf{s}_{i-1}+\mathbf{K}_{2} \mathbf{s}_{i+1} \\
& =\hat{\mathbf{M}}_{i} \ddot{\mathbf{q}}_{i r}+\hat{\mathbf{C}}_{i} \dot{\mathbf{q}}_{i r}+\hat{\mathbf{g}}_{i}\left(\mathbf{q}_{i}\right)-\mathbf{K}_{1} \mathbf{s}_{i}+\mathbf{K}_{2} \mathbf{s}_{i-1}+\mathbf{K}_{2} \mathbf{s}_{i+1}
\end{aligned}
$$

where $\mathbf{s}_{i}$ denotes the composite variable for the $i$-th robot such that $\mathbf{s}_{i}=\dot{\mathbf{q}}_{i}-\dot{\mathbf{q}}_{i r}$.

The parameter estimate $\hat{\mathbf{a}}_{i}$ for the $i$-th member is updated by the correlation integral: $\dot{\hat{\mathbf{a}}}_{i}=-\boldsymbol{\Gamma} \mathbf{Y}_{i}^{T} \mathbf{s}_{i}$, where $\boldsymbol{\Gamma}$ is a symmetric positive definite matrix. Hence, the closed-loop system for a network consisting of two non-identical robots can be written as $(i=1,2)$

$$
\left[\begin{array}{cc}
\mathbf{M}_{i}\left(\mathbf{q}_{i}\right) & \mathbf{0} \\
\mathbf{0} & \boldsymbol{\Gamma}^{-1}
\end{array}\right]\left(\begin{array}{c}
\dot{\mathbf{s}}_{i} \\
\tilde{\mathbf{a}}_{i}
\end{array}\right)+\left[\begin{array}{cc}
\mathbf{C}_{i}\left(\mathbf{q}_{i}, \dot{\mathbf{q}}_{i}\right)+\mathbf{K} & -\mathbf{Y}_{i} \\
\mathbf{Y}_{i}^{T} & \mathbf{0}
\end{array}\right]\left(\begin{array}{c}
\mathbf{s}_{i} \\
\tilde{\mathbf{a}}_{i}
\end{array}\right)=\left(\begin{array}{c}
\mathbf{u}(t) \\
\mathbf{0}
\end{array}\right)
$$

where $\mathbf{K}=\mathbf{K}_{1}+\mathbf{K}_{2}$ and $\mathbf{u}(t)=\mathbf{K}_{2}\left(\mathbf{s}_{1}+\mathbf{s}_{2}\right)$. Additionally, $\tilde{\mathbf{a}}$ denotes an error of the estimate such that $\tilde{\mathbf{a}}=\hat{\mathbf{a}}-\mathbf{a}$. Note that $\mathbf{a}$ is a constant vector of the true parameter values, resulting in $\dot{\tilde{\mathbf{a}}}_{i}=\dot{\hat{\mathbf{a}}}_{i}$.

Similar to Section IV, by applying the spectral transformation, we can show that the system is semi-contracting [3] with $\mathbf{K}_{1}-\mathbf{K}_{2}>0$ and $\mathbf{K}_{1}+\mathbf{K}_{2}>0$. Using Barbalat's 


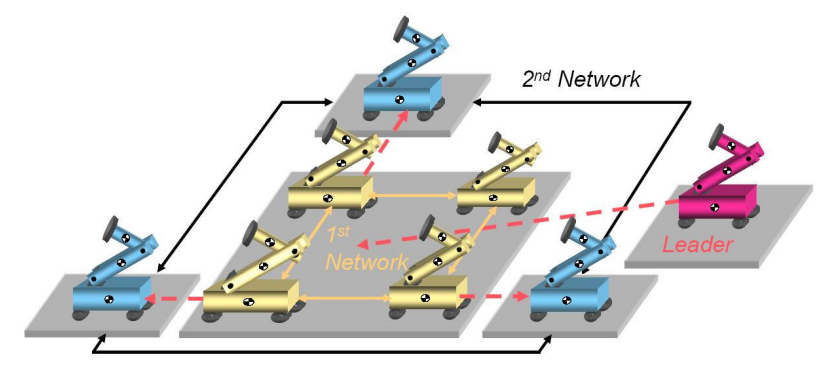

Fig. 5. Concurrent synchronization between two different groups. The desired trajectory inputs are denoted by the dashed-lines whereas the solid lines indicate mutual diffusive couplings. The independent leader sends the same desired trajectory input to the first network group.

lemma (see Section IV, Case II), it is straightforward to prove that $\mathbf{s}_{1}$ and $\mathbf{s}_{2}$ tend to each other asymptotically while the parameter estimates $\mathbf{a}_{1}$ and $\mathbf{a}_{2}$ synchronize as well.

\section{F. PD Synchronization of Robots}

One may consider the following Proportional and Derivative (PD) coupling control law for two identical robots from (1) for $(i=1, j=2)$ or $(i=2, j=1)$ :

$$
\tau_{i}=-\mathbf{K}_{1}\left(\dot{\mathbf{q}}_{i}+\boldsymbol{\Lambda} \tilde{\mathbf{q}}_{i}\right)+\mathbf{K}_{2}\left(\dot{\mathbf{q}}_{j}+\boldsymbol{\Lambda} \tilde{\mathbf{q}}_{j}\right)
$$

where the bounded reference position $\mathbf{q}_{d}$ has zero velocity such that $\dot{\tilde{\mathbf{q}}}_{i}=\dot{\mathbf{q}}_{i}$. Similar to Section IV-A, we can perform a spectral decomposition. By invoking LaSalle's invariant set theorem, we can conclude that $\mathbf{q}_{1}$ and $\mathbf{q}_{2}$ will follow $\mathbf{q}_{d}$ while $\mathbf{q}_{1}$ and $\mathbf{q}_{2}$ synchronize asymptotically. (see [1] for the detailed proof).

\section{G. Concurrent Synchronization}

In the context of the synchronization of multiple Lagrangian dynamics, discussed in this paper, we are interested in the concurrent synchronization [8] of different aggregates of multiple identical or nonidentical dynamics. As discussed earlier, we pay particular attention to the fact that there exist two different time scales of the proposed synchronization tracking control law. This in turn implies that there are two different inputs to the system, namely, the common reference trajectory $\mathbf{q}_{d}(t)$ and the diffusive couplings with the adjacent members. Accordingly, we exploit a desired trajectory $\mathbf{q}_{d}(t)$ to create multiple combinations of different dynamics groups. For instance, Figure 5 represents the concurrent synchronization of two different dynamical networks. The first network, consisting of four different robots, has the diffusive coupling structure proposed by the tracking control law in (2). The independent leader sends a desired trajectory command $\mathbf{q}_{d}$ to the first network. With an appropriate selection of gains, each dynamics in the first network synchronize while exponentially following the leader. Therefore, the proposed scheme can be interpreted in the context of the leaderfollower problem. The second network consists of three nonidentical dynamics, also different from those of the first group. Once the first network is synchronized, the second network also ends up receiving the same reference trajectory to follow while they interact to synchronize exponentially fast. Accordingly, we can achieve concurrent synchronization between two different network groups. This can be extended to arbitrarily large groups of synchronized dynamics by appropriately assigning the reference trajectory inputs and the diffusive couplings. Note that the number of agents in one layer can be different from that of another layer as seen in Figure 5.

\section{Conclusions}

This article has presented the new decentralized tracking control law that can be directly applied to synchronization and cooperative control of highly nonlinear robot dynamics. Providing exact nonlinear stability results constitutes one of the main contributions of this paper. Another benefit of synchronization is its implication for model reduction. It has been reported that the faster convergence rate represents the transient boundary layer dynamics of synchronization while the slower rate determines how fast the synchronized systems track the common reference trajectory. The exponential synchronization of multiple nonlinear dynamics allows us to reduce the dimensionality of the stability analysis of a large network. Further extensions to partial-joint coupling, PD coupling, time-delayed communications, adaptive synchronization and concurrent synchronization are also presented. For future work, we are interested in extending this methodology to dynamical networks on unbalanced or open graphs. Also, it would be useful to consider multi-agent systems consisting of underactuated dynamics.

\section{REFERENCES}

[1] Chung, S.-J., and Slotine, J.J.E, "Cooperative Robot Control and Synchronization of Lagrangian Systems," April 2007, submitted. Also, Chapter 5 of Soon-Jo Chung's Sc.D. thesis (MIT), 2007.

[2] Lin, Z., Broucke, M., and Francis, B., "Local Control Strategies for Groups of Mobile Autonomous Agents. IEEE Trans. on Automatic Control, 2004.

[3] Lohmiller, W., and Slotine, J.J.E., "On Contraction Analysis for Nonlinear Systems," Automatica, 34(6), 1998.

[4] Mesbahi, M., and Hadaegh, F.Y., "Formation Flying of Multiple Spacecraft via Graphs, Matrix Inequalities, and Switching," AIAA Journal of Guidance, Control, and Dynamics, (24) 2: 369-377, 2001.

[5] Olfati-Saber, R., and Murray, R.M., "Consensus Problems in Networks of Agents with Switching Topology and Time-Delays," in the special issue of the IEEE Transactions On Automatic Control on Networked Control Systems, VOL. 49, NO. 9, Sept. 2004.

[6] Ögren, P., Egerstedt, M. and Hu, X., "A Control Lyapunov Function Approach to Multiagent Coordination," IEEE Transactions on Robotics and Automation, Vol. 18, No. 5, October 2002.

[7] Ögren, P., Fiorelli, E., and Leonard, N.E., "Cooperative Control of Mobile Sensor Networks: Adaptive Gradient Climbing in a Distributed Environment," IEEE Trans. on Automatic Control, 49(8):1292.1302, 2004.

[8] Pham, Q.-C., and Slotine, J.J.E., "Stable Concurrent Synchronization in Dynamic System Networks," Neural Networks, 20(1), 2007.

[9] Rodriguez-Angeles, A., and Nijmeijer, H., "Mutual Synchronization of Robots via Estimated Sate Feedback: A Cooperative Approach," IEEE Transactions on Control Systems Technology, Vol. 12, No. 4, 2004.

[10] Wang, W., and Slotine, J.J.E., "On Partial Contraction Analysis for Coupled Nonlinear Oscillators," Biological Cybernetics, 92(1). 2004.

[11] Wang, W., and Slotine, J.J.E., "Contraction Analysis of Time-Delayed Communications Using Simplified Wave Variables," IEEE Trans. Aut. Control, 51(5), 2006. 